\title{
Improvement of Image Characteristics in Digital Breast Tomosynthesis by Incorporating a Compressed-sensing (CS) Deblurring Framework: Simulation Study
}

\author{
Heemoon $\mathrm{CHO}^{1}$, Kyuseok $\mathrm{KIM}^{2}$, Hyosung $\mathrm{CHO}^{3,}{ }^{*}$, Hyunwoo $\mathrm{LIM}^{4}$ \\ and Yeonok PARK ${ }^{5}$ \\ ${ }^{1}$ Department of Radiological Science, Yonsei University, 1 Yonseidae-gil, Wonju, \\ Gangwon-do 26493, Republic of Korea \\ ${ }^{2}$ Department of Radiological Science, Yonsei University, 1 Yonseidae-gil, Wonju, \\ Gangwon-do 26493, Republic of Korea \\ ${ }^{3}$ Department of Radiological Science, Yonsei University, 1 Yonseidae-gil, Wonju, \\ Gangwon-do 26493, Republic of Korea \\ ${ }^{4}$ Department of Radiological Science, Yonsei University, 1 Yonseidae-gil, Wonju, \\ Gangwon-do 26493, Republic of Korea \\ ${ }^{5}$ Department of Radiological Science, Yonsei University, 1 Yonseidae-gil, Wonju, \\ Gangwon-do 26493, Republic of Korea
}

${ }^{*}$ Corresponding author

Keywords: Digital breast tomosynthesis, Deblurring, Compressed-sensing.

\begin{abstract}
In this work, we considered a compressed-sensing (CS)-based framework with the total-variation regularization penalty for image deblurring of high accuracy in digital breast tomosynthesis (DBT). We implemented the proposed algorithm and performed a systematic simulation to demonstrate its viability for improving the image characteristics in DBT. In the simulation, blurred noisy projection images of a 3D breast phantom were generated by convolving their original (or exact) version by a $2 \mathrm{D}$ Gaussian blur kernel $(\sigma=2$ in pixel unit, kernel size $=11 \times 11)$, followed by adding Gaussian noise $\left(m=0, \sigma^{2}=0.05\right)$, and deblurred by using the proposed algorithm before performing DBT reconstruction.
\end{abstract}

\section{Introduction}

Digital breast tomosynthesis (DBT) is now considered in clinic as a standard x-ray imaging technique because it offers 3D mammographic acquisition with imaging dose comparable to standard 2D digital mammography (DM), overcoming the inherent shortcoming of the 2D DM [1]. In addition, DM is usually blurred and contaminated by noise through the imaging system, leading to relatively poor image characteristics, mainly due to the finite focal spot size of the x-ray tube, aspects inherent to detector such as the detector pixel size and resolution, and the system noise. Thus DBT images which are reconstructed from the acquired angle-dependent 2D mammographic images are also limited in the image performance. Fig. 1(a) shows the schematic illustration of a conventional DBT system in which a flat-panel detector remains stationary while an $\mathrm{x}$-ray tube moves in an arc during the projection data acquisition. Typical examination protocols in DBT acquire only 10 - 30 projection images taken over a limited angle range of $10^{\circ}-60^{\circ}$ and use a computationally-efficient filtered-backprojection (FBP) algorithm for DBT image reconstruction [2]. 


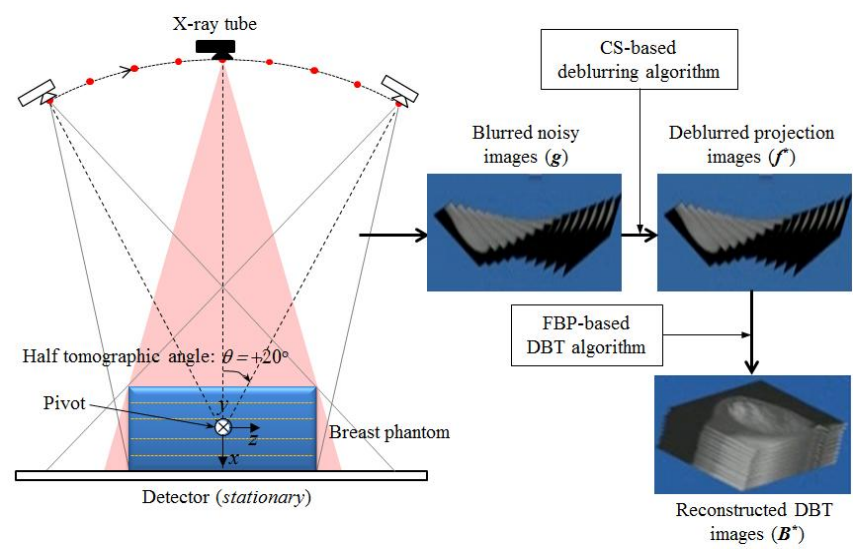

(a)

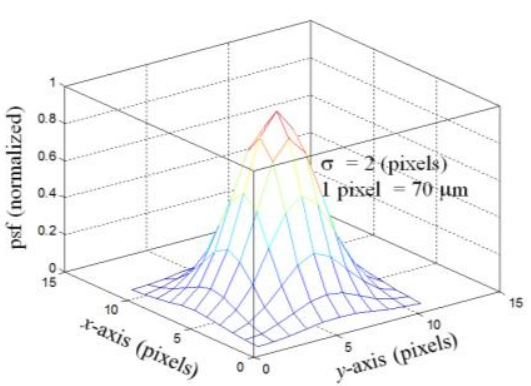

(b)

Figure 1. (a) Schematic illustration of a DBT system and the reconstruction procedure proposed in this study and (b) a perspective plot of the 2D Gaussian blur kernel designed for the image blurring in the simulation.

In this work, as an effective method to improve the image characteristics in DBT, we considered a compressed-sensing (CS)-based framework for image deblurring of high accuracy and incorporated it into the conventional DBT reconstruction. Here the CS is the state-of-the-art mathematical theory for solving the inverse problems, which exploits the sparsity of the image with substantially high accuracy [3]. We implemented the proposed algorithm and performed a systematic simulation to demonstrate its viability for improving the image characteristics in DBT.

\section{Material and Methods}

Most of the image deblurring methods are based on the standard image degradation model in which the observed (i.e., degraded) image, $g(x, y)$, is formed by convolving the original (or exact) image, $f(x, y)$, by the shift-invariant point-spread function (PSF), $p s f(x, y)$, of the system, followed by adding white Gaussian noise, $n(x, y)$, as follows:

$$
g(x, y)=f(x, y) * * \operatorname{ps} f(x, y)+n(x, y) .
$$

Here the operator $* *$ represents $2 \mathrm{D}$ convolution and the $\operatorname{psf}(x, y)$ is the $2 \mathrm{D}$ profile of the image of a point object which describes the amount of blurring by the imaging system. Thus the degraded image can be restored by the deconvolution scheme, provided that the PSF is known. However, as indicated in Eq. (1), one difficulty in the deconvolution problem is associated with the presence of noise in the degraded image, which often results in an unstable or false solution. The typical counter-measure to this problem is the introduction of a regularization penalty term which controls the compromise between spatial resolution and noise in the image.

In this work, as a highly accurate deconvolution scheme, we considered a CS-based deblurring scheme which is incorporated with the total-variation (TV) regularization penalty. Here the TV is the $l^{1}$-norm of the gradient image and its minimization strategy is incorporated into the CS-based framework [4]. In the CS-based deblurring scheme, the original image $f$ is recovered normally by minimizing the following objective function, $(f)$, as the solution to a convex optimization problem, $f^{*}$, described in Eq. (2), assuming that the negligible components of the gradient image are zeros [5]: 


$$
\begin{aligned}
& f^{*}=\underset{f \in Q}{\arg \min } \phi(f), \\
& \phi(f)=\frac{1}{2}\|f * * p s f-g\|_{2}^{2}+\alpha\|f\|_{T V},
\end{aligned}
$$

where $Q$ is the set of feasible $f, 1 / 2\|f * * p s f-g\|_{2}^{2}$ is the fidelity term, $\|f\|_{T V}$ is the TV regularization penalty term, and $\alpha$ is the parameter that balances the two terms and is chosen so that signal-to-noise ratio is maximized (e.g., $\alpha=0.1$ was used in the simulation). Detailed descriptions of the FBP-based DBT algorithm incorporated with a CS-based deblurring framework can be found in our previous paper elsewhere [6].

Based on the above descriptions, we implemented the proposed algorithm and performed a systematic simulation. In the simulation, all the projection images were taken with a half tomographic angle of $\theta=20^{\circ}$ and an angle step of $\Delta \theta=2^{\circ}$. The resultant DBT images were reconstructed with a voxel size of $116 \mu \mathrm{m}$, a voxel dimension of $250 \times 190 \times 280$, and a pixel size of $70 \mu \mathrm{m}$. The number of iterations in the CS-based deblurring scheme was set to 150 because the convergence rate of the algorithm seemed to be saturated after about 100 iterations. Fig. 1(b) shows the perspective plot of the 2D Gaussian blur kernel ( $\sigma=2$ in pixel unit, kernel size $=11 \times$ 11) designed for image blurring in the simulation.

\section{Results and Discussion}

Fig. 2(a) shows some examples of (a) the original (top), the blurred noisy (middle), and the resultant deblurred projection images (bottom) of the breast phantom. Only the three projection images out of 21 are indicated for simplicity. The images on the extreme-right column are the enlarged images inside the box indicated in Fig. 2(a). Note in Fig. 2(a) how the deblurred image is quite clear, compared to the blurred image, indicating the effectiveness of the proposed deblurring scheme for improving the image characteristics in 2D DM at least qualitatively. Fig. 2(b) shows some examples of the phantom slice images (top) and the reconstructed DBT images (middle \& bottom) of

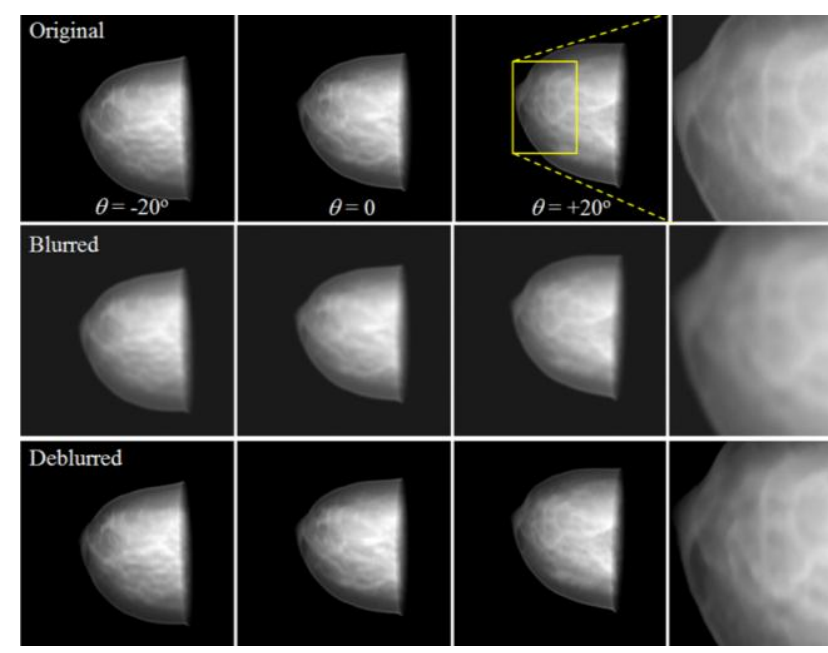

(a)

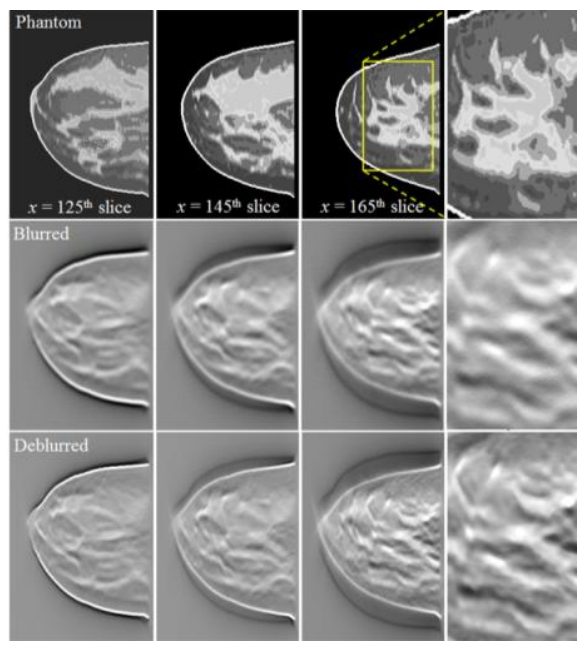

(b)

Figure 2. Some examples of (a) the original (top), the blurred noisy (middle), and the resultant deblurred projection images (bottom), and (b) the phantom slice images (top) and the reconstructed DBT images (middle \& bottom) of the 3D breast phantom before and after applying the proposed deblurring framework, respectively. 
the breast phantom before and after applying the proposed deblurring framework, respectively. The corresponding phantom images are also indicated as the reference. Fig. 2(b) shows some examples of the phantom slice images (top) and the reconstructed DBT images (middle \& bottom) of the breast phantom before and after applying the proposed deblurring framework, respectively. The corresponding phantom images are also indicated as the reference. The images on the extreme-right column are the enlarged images inside the box indicated in Fig. 2(b), indicating the effectiveness of the proposed deblurring scheme for improving the image performance in DBT as well as 2D DM.

\section{Summary}

In this work, as a continuation of our mammography R\&D, we investigated a CS-based deblurring scheme incorporated with TV regularization penalty for image deblurring of high accuracy and adopted it into the image reconstruction in conventional DBT. We implemented the proposed algorithm and performed a systematic simulation to demonstrate its viability for improving the image performance in DBT. Our simulation results indicate that the proposed deblurring scheme seems effective for improving the image performance in DBT as well as 2D DM. We expect the proposed deblurring algorithm to be applicable for improving the present image characteristics in standard 2D mammography and DBT systems in clinics.

\section{Acknowledgement}

This work was financially supported by the Radiation Technology Development Program of the National Research Foundation (NRF) funded by the Korea Ministry of Science, ICT \& Future Planning under Contract No. 2015-51-0284.

\section{References}

[1] S. Ciatto, N. Houssami, D. Bernardi, F. Caumo, M. Pellegrini, S. Brunelli, et al., Integration of 3D digital mammography with tomosynthesis for population breast-cancer screening (STORM): a prospective comparison study, Lancet Oncol. 14 (2013) 583-589.

[2] M. A. Helvie, Digital mammography imaging: breast tomosynthesis and advanced applications, Radiol. Clin. North Am. 48 (2010) 917-929.

[3] K. Choi, J. Wang, L. Zhu, T. S. Suh, S. Boyd, L. Xing, Compressed sensing based cone-beam computed tomography reconstruction with a first-order method, Med. Phys. 37 (2010) 5113-5125.

[4] S. Babacan, R. Molina, A. Katsaggelos, Parameter estimation in TV image restoration using variational distribution approximation, IEEE Trans. Image Process. 17 (2008) 326-39.

[5] H. Takeda, S. Farsiu, P. Milanfar, Deblurring using regularized locally adaptive kernel regression, IEEE Trans. Image Process. 17 (2008) 550-563.

[6] J. E. Oh, H. S. Cho, D. S. Kim, S.I. Choi, U. K. Je, Application of digital tomosynthesis (DTS) of optimal deblurring filters for dental x-ray imaging, J. of the Korean Phys. Soc. 60 (2012) 1161-1166. 